Hugo van Woerden

Kapteyn Astronomical Institute

Groningen, The Netherlands

As mentioned already in Morton Roberts' fine introduction, two features were striking in this Symposium's programme: the broad spectral range of observational data, and the strong emphasis on comparisons with external galaxies. I shall return to the latter item towards the end of my summary. Let us first review the spectral panorama, then scrutinize what it has taught us about our Galaxy.

\title{
THE SPECTRAL PANORAMA
}

For the first time in this series of symposia about our Galaxy, gamma-ray studies made significant contributions: Paul discussed the $\gamma$-ray disk, thin as that of the gas; and Stecker the $\gamma$-ray halo, more properly perhaps called a thick disk. X-rays and ultraviolet were less prominent now than five years ago, at IAU Symposium 60 .

Optical observations contributed some of the highlights of this Symposium. First and foremost, Vera Rubin's beautiful rotation curves of external galaxies gave strong evidence for extended mass distributions and demonstrated density-wave effects in the differential motions across spiral arms. The fine photographic $\mathrm{H} \alpha$ surveys of Sivan and the Georgelins testify to the wide spread of ionized hydrogen, and provide ring diameters - of key importance for determinations of the extragalactic distance scale. Spectral studies of HII regions (Peimbert) show that abundances of heavier elements decrease with increasing galactocentric distance. Abundances also come from stellar spectra (Kraft). Indispensable, too, are stars as distance indicators in studies of spiral structure (Roberta Humphreys) and size of the Galaxy (Graham). Our only estimate of the Galaxy's morphological type (by De Vaucouleurs) was based on photometric work.

In the infrared, Fabry-Pérot studies of the [NeII]-1ine have given new, deep insights into the galactic nucleus (Wollman). The far infrared (Mezger, Puget) is our best probe of the dust clouds in the inner regions, and their role in star formation.

591

W. B. Burton (ed.), The Large-Scale Characteristics of the Galaxy, 591.598.

Copyright $\odot 1979$ by the IAU. 
Observationally, the Co-surveys at $2.6 \mathrm{~mm}$ (Burton, Liszt, Bania, Solomon, Scoville) may represent the most massive advance since 1973 . Our views of the whole "inner half" of the Galaxy have been changed drastically by this new tool. Thus, $\mathrm{H}_{2}$ molecules are now thought to rival or surpass HI atoms as the major constituent of the interstellar gas. The high angular resolution in this millimeter line has caused severe undersampling in the major surveys, but this situation is now improving considerably (R.S. Cohen). CO is also being developed as a tracer of the molecular gas in other galaxies (Rickard). Potentially, the CO-lines could become as important as the $21-\mathrm{cm} H I-1$ ine for studies of distribution, kinematics and dynamics of interstellar gas in external gal axies.

A recent addition to the spectrum is the $\mathrm{CH}-1$ ine at $9 \mathrm{~cm}$. Johansson presented results of the first survey of this widespread molecule. At centimeter wavelengths, we must further mention the massive surveys of $\mathrm{H}_{2} \mathrm{O}, \mathrm{H}_{2} \mathrm{CO}$, recombination 1 ines and continuum carried out at Effelsberg (Downes).

The hydrogen line at $21 \mathrm{~cm}$ has been a major galactic research tool since 1951. At this Symposium, it brought new views on the Galactic Centre (Burton, Liszt, R.J. Cohen), on the physics of the interstellar medium (Salpeter, Baker, Crovisier), on the rotation, mass and size of the Galaxy (Gill Knapp, Jackson), and on its environment (Hulsbosch, Giovanelli, Mathewson). In particular also, hydrogen-line studies of external galaxies at resolutions $\leqslant 1 \mathrm{kpc}$ have a strong impact on our thoughts about properties of our Galaxy.

Long radio waves play a minor role in galactic radio astronomy nowadays. It is ironic that the galactic halo, discussed at IAU Symposium 5 in 1955 on the basis of meter wave observations, was now the subject of gamma-ray studies.

Strong improvements in the panorama are required at gamma- and IRwavelengths, and in the $\mathrm{CO}-$ and $\mathrm{CH}-1$ ines. And, of course, optically we still know our Galaxy very little, though there is good hope for more: 0 -stars at $50 \mathrm{kpc}$ distance have been reported.

\section{THE GALAXY UNDER SCRUTINY}

What then have all these photons, large and small, told us about the large-scale characteristics of our Galaxy?

Nucleus and Central Region $(\mathrm{R}<4 \mathrm{kpc})$

Let us start at the smallest structural unit, the nucleus. As noted by Oort, VLBI results (not reported at this Symposium) give evidence of an 0":001-diameter core source. Virginia Trimble has summarized the views of Paczyński and Ozernoj on what might reside or be missing within those nuclear light-hours, and supplemented them with her own thoughts. 
Wollman's 12.8- $\mu$ m NeII observations have revealed a $10^{6} .5 \mathrm{M}_{\odot}$ concentration within 1 pc diameter; the dynamical centre coincides with IRS 16, the peak of the infrared nuclear bulge. This galactic core contains, within the Sgr A molecular complex, a disk of HII, rotating and expanding at $200 \mathrm{~km} / \mathrm{s}$, and tilted $45^{\circ}$ to the galactic plane. Whether this dust-filled HII-region (Mezger) produces young stars, is under debate.

Within $150 \mathrm{pc}$ of the Centre, 6-cm continuum observations (Mezger) show $10^{6} \mathrm{M}_{\odot}$ of extended, low-density $\left(10 \mathrm{~cm}^{-3}\right) \mathrm{HII}$, plus several giantHII-regions caused by massive star formation. The molecular ring at $\mathrm{R}=200 \mathrm{pc}$ (Scoville, Sanders et al.) now is viewed as part of a tilted nuclear disk. Burton and Liszt have shown that a thin disk, of $1.5 \mathrm{kpc}$ radius, $\sim 25^{\circ}$ tilt and with rotating and expanding motions, can represent all sofar isolated features in one unified model; the disk contains $10^{7} \mathrm{M}_{\odot}$ of $\mathrm{HI}, 10^{9-10} \mathrm{M}_{\odot}$ of $\mathrm{H}_{2}$ gas, and $10^{10.4} \mathrm{M}_{\odot}$ of stars.

Sanders has derived a bulge rotation curve from the $2-\mu \mathrm{m}$ IR brightness distribution. This curve nicely fits to the $21-\mathrm{cm}$ disk rotation curve; also, the bulge may be viewed as the core of a $5 \times 10^{10} \mathrm{M}_{\odot}$ halo. The double-peaked rotation curve allows an explanation of the gas deficit at $1<\mathrm{R}<4 \mathrm{kpc}$ in terms of angular-momentum transfer through turbulent-shear viscosity. This explanation differs fundamentally from that proposed by Oort (IAU Symposium 58), who ascribed the gas deficit to exhaustive star formation induced by frequent density-wave passages. Sanders further represents the noncircular motions of the 3-kpc arm ("Rougoor's arm", I prefer to call it) by motion along elliptical streamlines around a slight oval distortion in the mass distribution.

As wishes for further work in these regions, I note: multi- $\lambda$ IR maps of the inner core, and the dynamics of the various tilted-disk features.

\section{The 5-kpc Ring: Molecules and Star Formation}

To an outside observer, the region $3<\mathrm{R}<8 \mathrm{kpc}$ (assuming still $R_{O}=10 \mathrm{kpc}$ for the Sun-Centre distance) would be the dominant "Population-I" feature in our Galaxy. Here we find the peaks of the radial distributions of molecules, HII-regions, supernova remnants (SNR), pulsars, and gamma-ray sources. As noted by Burton and Gordon in the 1976 Annual Reviews, only HI deviates from this.

Extensive new surveys (Solomon) place the CO peak at $R=5.5 \mathrm{kpc}$. The vertical distribution peaks at $z_{0} \sim-30 \mathrm{pc}$, the layer thickness (FWHM) is $\sim 120 \mathrm{pc}$. The gas is concentrated in "clouds" of $50 \mathrm{pc}$ size, density $2 \mathrm{H}_{2} \mathrm{~cm}^{-3}$ and mass $10^{5} \mathrm{M}_{\odot}$; in toto, our Galaxy would contain $4 \times 10^{9} \mathrm{M}_{\odot}$ of $\mathrm{H}_{2}$. These densities and masses are based on an assumed density ratio ${ }^{13} \mathrm{CO} /{ }^{12} \mathrm{CO} / \mathrm{H}_{2}=0.5 / 20 / 10^{6}$. As noted by Peimbert, the abundance ratio $\mathrm{C} / \mathrm{O}$ varies with $\mathrm{R}$, hence the ratio $\mathrm{H}_{2} / \mathrm{CO}$ is probably overestimated. Can we find effective ways to observe $\mathrm{H}_{2}$ directly? 
A pressing theoretical problem is that of support of the molecular clouds against gravitational collapse (Solomon, Turner). The spatial COdistribution suggests that clouds live $10^{8-9}$ yr rather than form stars at a rapid rate. Scoville speculates that they grow by collision.

The Onsala Group is to be congratulated on their first CH..survey (Johansson). With a longitude spacing of 2.5 and $5^{\circ}$, this work is at the same stage as HI in 1953. Already, it is clear that the $\mathrm{CH}-1$ ines trace the diffuse component of the molecular gas, including warm gas, while the CO-lines favour the dense, cool clouds. The z-distribution of $\mathrm{CH}$ resembles that of $\mathrm{CO}$, but its radial range $(4-10 \mathrm{kpc})$ is greater. Davies notes that $\mathrm{H}_{2} \mathrm{CO}$ represents an intermediate-density regime between $\mathrm{CO}$ and $\mathrm{CH}$. The physics of molecular clouds was reviewed in detail by Turner.

Lockman finds peaks at $R=5$ and $7 \frac{1}{2} \mathrm{kpc}$ in the radial distribution of HII-regions. The vertical distribution is the thinnest known: $\sigma(z) \sim$ $30 \mathrm{pc}$. Of great interest is the "ripple" in the run of $z$ with $R$, a "corrugation" in the galactic plane, also found in HI, CO and SNR.

I mentioned earlier the beautiful $\mathrm{H} \alpha$ surveys of Sivan and Georgelin. Another phantastic atlas is that of Downes et al., who mapped a $2^{\mathrm{O}} \times 62^{\mathrm{O}}$ region along the galactic equator at $6 \mathrm{~cm}$ with $2: 6$ resolution, using the Effelsberg telescope. For 1000 sources, kinematic distances were obtained from $6-\mathrm{cm}$ line studies. This is a giant jump ahead in the mapping of HII regions.

\section{Local Interstellar Physics}

The region $R \sim 10 \mathrm{kpc}$ is where we know most about structure and physical conditions in the interstellar medium. Forty years ago, Strömgren distinguished HII-regions and HI-clouds. Ten years ago, another two-component model: clouds and intercloud medium (ICM), was inwented by Pikel'ner, Spitzer, Field and associates. Recently, a hot ionized component (traced by OVI lines) was added to the three proposed earlier: warm ionized, warm neutral, and cold neutral gas. This week, Salpeter added molecular clouds, lukewarm clouds, interfaces, and coronal gas, bringing the total to 8 "phases". And Turner distinguishes 6 types of molecular clouds: At this pace, the next symposium on interstellar physics may well see a classification scheme for interstellar structures as detailed as current ones for galaxies and even stars. Nevertheless, a special meeting to review the observational evidence for, and physical processes in, these various phases might well be worthwhile. Salpeter already has given estimates of mass flux, energy balance etc. It struck me that he considers most of the electrons to be inside HII-regions rather than in the (late?) ICM.

Baker discussed the physical processes in cold, warm and hot neutral hydrogen. Important was his emphasis on the strong effects that sidelobe responses ("stray radiation") have on $21-\mathrm{cm}$ line profiles at high galactic latitudes. These stray radiation contributions have 
strongly affected both ICM parameters and studies of correlation between gas and dust. I further recall his remarks about the transient nature of interstellar structures such as filaments and sheets: " interstellar weather". Gone are the days of spherical standard clouds ...

About the motions of these structures, much information is contained in the new Nançay Survey of $21-\mathrm{cm}$ absorption in $\sim 1000$ source spectra (Crovisier). The numbers are similar to those of earlier surveys: $\sigma \sim 6$ $\mathrm{km} / \mathrm{s}$ for external, $0-3 \mathrm{~km} / \mathrm{s}$ for internal motions, but the data base is far broader.

Weaver discussed the relationship between continuum loops and supernova remnants. He ascribes the North Polar Spur to a SN explosion inside a giant bubble blown by the Sco-Cen cluster. Heiles showed us supershells of kiloparsec size, due to $10^{53}$-erg explosions that no astronomer has yet seen or photographed, here or far out in extragalactic space.

\section{Spiral Structure}

We have seen maps showing the distributions of $\mathrm{HI}$, HII-regions (optical: stellar distances, and radio: kinematical), giant-HII's, $\mathrm{H}_{2} \mathrm{CO}$ and $\mathrm{CO}$ molecular clouds, young stars, clusters and Cepheids. There is no full agreement among these maps, but Georgelin's 4-armed pattern seems to have a strong case. Given the observational evidence and theoretical case for noncircular motions, the direct stellar distances of Roberta Humphreys are of great value. An important need is for a wider range of such distances. Can we find suitable (infra)red, young objects?

Wielen has given a lucid review of various aspects of density-wave theory. I recall his remark that only old objects such as stars and HI gas can show the waves (with shocks for the gas), while the distribution of young objects (including HII-regions) is determined by migration from their birth places near the shock-front. The migration depends on the initial velocities (pre- or post-shock?); predictions are fairly complex, and comparison with observations difficult. Comparison of the densitywave predictions with observations has been very successful for HI in M81 (Visser). A similar confrontation of shock theory and observations for HI in our Galaxy would be of major interest.

Another important point in Wielen's paper is his discussion of average motions and the local standard of rest. It seems that our Symposium Chairman, Kerr, was closer to truth in 1962 than many of us believed.

Woodward's calculations of star formation in galactic shocks suggest a possible process that starts at one end, and then propagates through a cloud. Bash described calculations of cluster evolution after formation in a density wave; he can represent disk colours and brightness profile. 


\section{Galactic Rotation and Integral Properties}

Both Vera Rubin, from optical spectra, and Albert Bosma, from HI syntheses, conclude that rotation curves are flat in the great majority of galaxies; hence, there is much mass in the outer parts, the M/L ratio must increase outward, and masses approaching $10^{12} \mathrm{M}_{\odot}$ now appear 1 ikely in many giant galaxies. From HI observations in our Galaxy, Jackson also finds a flat rotation curve, with large-scale regional deviations; at $12<\mathrm{R}<14 \mathrm{kpc}, \mathrm{d} \theta / \mathrm{dR}>0$, suggesting a density-wave effect across the Perseus Arm. Note that, on a rippled rotation curve, the Oort constants $A$ and $B$ have only local meaning. We have been lucky for 50 years not to have been led astray by them!

Although Einasto and Gill Knapp propose a local rotation speed of $220 \mathrm{~km} / \mathrm{s}$, and $\mathrm{R}_{\mathrm{O}}=8.5 \mathrm{kpc}$, the latter finds flat rotation to such great distances that our Galaxy, too, must be placed in the 100-kpc diameter, $10^{12}-M_{\odot}$ class. An interesting related point is that the velocity of escape becomes much higher $(550 \mathrm{~km} / \mathrm{s}$, see 0striker) than we believed some time ago. Meanwhile, there is enough dispute about these rotation parameters, and their effects on the fate of Magellanic Clouds and Stream, that a workshop to study these problems in detail might be fruitful.

While the case for flat rotation curves and consequent high masses appears very strong, one must realize that small errors in the derived curves - such as may be caused by noncircular motions and by disk warps - may have strong effects in the masses. It is thus important to supplement such mass determinations with other methods, especially: measurement of relative motions in galaxy pairs.

De Vaucouleurs has presented us with an impressive list of parameters for our Galaxy. On many of these parameters the errors are so small that I have wondered whether a trip far outside the galactic plane would still pay.

\section{Rim, Fringes, Loose Ends, etc.}

Henderson has shown new data on the shape and thickness of the galactic gas layer in its outer parts. In fact, although Habing, Kepner, Davies, Verschuur and others had drawn attention to the large vertical extent of these outer parts, no complete, systematic mapping had been done since the fifties. Henderson finds a warp of $z_{0}=1.6 \mathrm{kpc}$ at $\mathrm{R} \sim$ $1.7 \mathrm{R}_{\mathrm{O}}$, and $1.5 \mathrm{kpc}$ disk thickness along the outer edge of the Perseus Arm.

Warps stronger than ours are frequent among external galaxies, as discovered by Sancisi at Westerbork, and new theoretical work was overdue. Saar's review showed that massive haloes of various shapes can help in various ways: spherical haloes can facilitate tidal forcing, triaxial haloes can produce unstable oscillations, and in elliptical haloes 
accretion may lead to bending and thickening of the gas layer. Tubbs and Sanders demonstrated that both primordial and tidal warps can persist for $5 \times 10^{9}$ yr in galaxies where the halo mass exceeds that of the disk, within the latter's radius. A prediction that invites further observational test is that these warps should develop only in the tenuous gas outside the (truncated!) stellar disk. Further observation also should strengthen the statistics of warps, and reveal their shapes farther out.

The nature of high-velocity clouds has been the subject of fierce debate for many years. I was happy to hear Verschuur acknowledge that the name HVCS may stand for a variety of phenomena of different nature. Hulsbosch and Giovanelli have made new, extremely sensitive surveys at velocities out to $\pm 1000 \mathrm{~km} / \mathrm{s}$; both find clouds with velocities down to $-465 \mathrm{~km} / \mathrm{s}$, and their results agree in detail. The distance and nature of these objects is unclear, though a suggestion prevails that they may be intergalactic hydrogen clouds in the Local Group. In fact, some may be related to the Magellanic Stream.

Mathewson and Fujimoto summarized observations and theory of the Magellanic Stream. So far, nothing but HI has been observed in the Stream, but its "magellanic" distance and "overhead" geometry appear secure. No satisfactory model has yet been developed, but it seems likely that we must interpret the Stream as a tidal effect, caused in the Magellanic Clouds by a high-mass $\left(\geqslant 5 \times 10^{11} \mathrm{M}_{\odot}\right)$ galaxy. The Cloud pair would then be in grave danger of disruption (a fact to be regretted by Bart Bok), and our warp have to be blamed on non-magellanic causes.

Martha Haynes presented a convincing case that isolated intergalactic hydrogen clouds outside the Local Group have sofar eluded detection. What intergalactic gas has been observed in other galaxy groups can be properly described as tidal debris. To me it seems, however, that such debris must dissipate; does it then become unobservable?

\section{Formation and Evolution}

Our collective scrutiny of the Galaxy has been strongly descriptive - both as regardsobservations and theoretical models. Few papers only discussed how its present structure developed. However, Beatrice Tinsley's impressive discussion of the formation and evolution of galaxies well made up for this. One item I mention: not only high-velocity clouds, or even the warp of the Galaxy's disk, may be due to accretion; the disk itself may have been accreted rather than be primordial.

\section{THE GALAXY? -}

Throughout this symposium, detailed information on external galaxies has served not only as a comparison for our Galaxy, but indeed to guide our understanding. I note Strom's massive review of the light and mass distribution, dynamics and evolution (chemical and morphological) of 
bulge and disk components. I recall again the optical and radio rotation curves and mass distribution, and the demonstration of density-wave theory. I further mention Rots' summary of the distributions of atomic and molecular hydrogen and of continuum radiation. Weedman has shown how minute our Galaxy's "nuclear activity" is by extragalactic standards. It appears obvious that our quest for understanding of the origin of spiral structure and of the Galaxy itself will ultimately be answered from the realm of the nebulae.

This emphasis on comparisons with other galaxies is a sign that galactic research has grown up. We, inhabitants of our Galaxy, have learned: "know thy neighbours in order to understand thyself". But this does not say that galactic research will die, now that we have proper tools for research into galaxies. In fact, only at home can we determine three-dimensional kinematics and distribution. And it is here that we can go ten magnitudes fainter (minus wavelength-dependent extinction) and achieve 100 times better linear resolution than even in Andromeda.

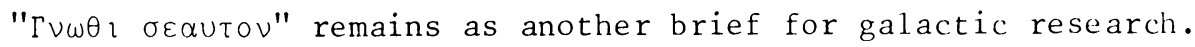

These two adagia may strike you as philosophical or anthropological rather than astronomical. However, our science - as any other - is and remains a human enterprise. One of the great joys of astronomy is its strongly international character. In this connection, it is sad that this Symposium's audience was geographically so poorly balanced. Asia and - alas - Africa were badly underrepresented. So were South America and - especially - Australia, whose powerful telescopes have such a unique job to do in the southern hemisphere. Also, we have sorely missed our colleagues from Eastern Europe, particularly the Soviet Union. Finally, I regret the small representation of my home country with its strong galactic tradition. Has Westerbork drawn us too far away from our Galaxy? May the next galactic symposium be more truly international:

\section{Acknowledgements}

I wish to thank the organizers, and especially Frank Kerr, for a most stimulating Symposium. Many speakers have contributed to this written version of my off-the-cuff summary by sending preprints of their papers. 